\title{
High Resolution Zero Degree Electron Spectroscopy of Argon Ions through Carbon Foil
}

\author{
M. Imai ${ }^{1}$, M. Sataka ${ }^{2}$, K. Kawatsura ${ }^{3}$, K. Takahiro ${ }^{3,4}$, K. Komaki ${ }^{5}$, and H. Shibata ${ }^{1}$ \\ ${ }^{1}$ Department of Nuclear Engineering, Kyoto University, Sakyo, Kyoto 606-8501, Japan \\ ${ }^{2}$ Department of Materials Science, Japan Atomic Energy Research Institute, Tokai, Ibaraki 319-1195, Japan \\ ${ }^{3}$ Department of Chemistry and Materials Technology, \\ Kyoto Institute of Technology, Sakyo, Kyoto 606-8585, Japan \\ ${ }^{4}$ II. Institute of Physics, University of Göttingen, Friedrich-Hund-Platz 1, D-37077 Göttingen, Germany \\ 5 Institute of Physics, Graduate School of Arts and Sciences, University of Tokyo, Meguro, Tokyo 153-8902, Japan
}

Received on 29 July, 2005

\begin{abstract}
Electrons emitted from high Rydberg states formed by penetration of $2.0 \mathrm{MeV} / \mathrm{u} \mathrm{Ar}^{6,13,14,15+}$ ions through C-foil targets of various thicknesses $\left(4.0-20 \mu \mathrm{g} / \mathrm{cm}^{2}\right)$ have been measured with high resolution utilizing zero-degree electron spectroscopy. Intense series of Coster-Kronig peaks due to $\operatorname{Ar}^{14+} 1 \mathrm{~s}^{2} 2 \mathrm{p}\left({ }^{2} \mathrm{P}_{3 / 2,1 / 2}\right) n l$ $1 \mathrm{~s}^{2} 2 \mathrm{~s}\left({ }^{2} \mathrm{~S}_{1 / 2}\right) \varepsilon l^{\prime}$ and $\mathrm{Ar}^{13+} 1 \mathrm{~s}^{2} 2 \mathrm{~s} 2 \mathrm{p}\left({ }^{3} \mathrm{P}_{2,1,0}\right) n l-1 \mathrm{~s}^{2} 2 \mathrm{~s}^{2}\left({ }^{1} \mathrm{~S}_{0}\right) \varepsilon l^{\prime}$ transitions, in which the principal quantum number, $n$, starts at 10 and increases with increasing peak energy, have been observed for every combination of initial charge state and foil thickness, and quite a few number of other peaks remain unassigned in the electron energy region investigated. Intensities of each series of Coster-Kronig electron peaks follow the $n^{-3}$ law. The mean charge states after the foil penetration have been found to be the same within $10 \%$ uncertainty and the total intensity of the Coster-Kronig transitions including $\mathrm{Ar}^{15+} 1 \mathrm{~s}^{2} 2 \mathrm{p}\left({ }^{2} \mathrm{P}\right)$ and $\mathrm{Ar}^{14+} 1 \mathrm{~s}^{2} 2 \mathrm{~s} 2 \mathrm{p}\left({ }^{3} \mathrm{P}\right)$ cores have also been found to remain constant for all the collision systems investigated. The total $\mathrm{Ar}^{15}+1 \mathrm{~s}^{2} 2 \mathrm{p}\left({ }^{2} \mathrm{P}\right)$ population derived from $n=10$ transition peaks is found to be almost the same as those of $\mathrm{Ar}^{14+} 1 \mathrm{~s}^{2} 2 \mathrm{~s} 2 \mathrm{p}\left({ }^{3} \mathrm{P}\right)$, which is in contrast to the ratio of $\mathrm{Ar}^{15+}$ to $\mathrm{Ar}^{14+}$ fractions measured downstream the foil.
\end{abstract}

Keywords: Rydberg state; Coster-Kronig transition; Charge state; Density effect

\section{INTRODUCTION}

Dynamic properties of the collision processes inside a solid target have been one of the major interests in ion-solid interactions and the penetration of ions through solid target has been investigated in many aspects. A cusp-shaped secondary electron peak in the forward direction and a series of electron peaks emitted from Rydberg states of fast projectile can also be observed in much simpler ion-gas collisions. In the ionsolid collision, on the contrary, more intense series of CosterKronig (C-K) electron peaks from Rydberg states have been observed than expected by Coulomb capture of target electron or by bound-bound excitation of projectile electrons at the last layer collisions on the exit surface, although the Rydberg state can not survive inside the solid because the orbital radius of Rydberg electron is far larger than the lattice spacing of the solid target [1]-[3]. This indicates that the Rydberg states are formed upon or near the exiting surface of the solid, capturing electrons which have been accumulated to follow the projectile at the same velocity by being released from projectile bound states or captured from the target during foil penetration. A model for producing such Rydberg or convoy electrons has been introduced by Rothermel et al. [1] and concluded by Burgdörfer and Bottcher [4] as a three-step transport model. In their model, electrons in near-threshold projectile continuum move at the projectile velocity while inside solids and, upon leaving the foil, a part of such electrons are re-captured to form the Rydberg states, shaken by a sudden switch of projectile potential from screened to bare Coulomb potential. Consequently, the formation of the Rydberg states is inferred to be strongly related to that of the "cusp" elec- trons, depending on whether the electron is captured to bound states or stays on the continuum state.

So far the zero-degree electron spectroscopy [5],[6] has been proven to be a powerful tool to study these Rydberg state ions consisting of the excited ion core and Rydberg electron, through observing the $\mathrm{C}-\mathrm{K}$ electrons ejected at zero degrees with high resolution. Another advantage in precise measurements of $\mathrm{C}-\mathrm{K}$ electron energy is that the ion configurations inside the solid can partly be probed by identifying the core configurations of the observed Rydberg states. Moreover, as $\mathrm{C}-\mathrm{K}$ electron energy can be determined free from contact potentials of the surrounding equipments by zero-degree electron spectroscopy, non-equilibrium collision phenomena can be investigated using a very thin foil as target.

We have been studying the electron emission mechanisms from various ions with different energies passing through gas and solid targets with zero-degree electron spectroscopy [7][12]. As for solid target, we have studied S and Si projectile ions, to prove that the decays of 3-5 electron systems give a significant contribution in the electron spectrum for 2.0 $\mathrm{MeV} / \mathrm{u} \mathrm{S}^{12+}$ ion penetrating through a $2.2 \mu \mathrm{g} / \mathrm{cm}^{2}$ carbon foil [7],[10], and so do those of 4-5 electron system in $2.0 \mathrm{MeV} / \mathrm{u}$ $\mathrm{Si}^{5+}$ ion through a $9.6 \mu \mathrm{g} / \mathrm{cm}^{2}$ carbon foil target [8] by using $\mathrm{C}-\mathrm{K}$ electron energies calculated by perturbation theory of $Z$-expansion method ( $\mathrm{MZ}$ code). We reproduced the experimental spectra fairly well by convoluting the calculated spectra with the spectrometer resolution and the ion charge population "inside" the foil [9],[10]. We have extended our work to other projectiles in order to study the formation of Rydberg ions systematically, and present here results for 2.0 $\mathrm{MeV} / \mathrm{u} \mathrm{Ar} 6,13,14,15+$ ions traversing $4.0-20 \mu \mathrm{g} / \mathrm{cm}^{2}$ carbon 
foil targets.

\section{EXPERIMENTS}

The present experiments were performed at the tandem accelerator facility at the Japan Atomic Energy Research Institute (JAERI), Tokai. As the detailed description of the experimental apparatus has been presented previously [7]-[12], only some major parameters are given here. A beam of 80 $\mathrm{MeV}(2.0 \mathrm{MeV} / \mathrm{u}) \mathrm{Ar}^{6+}$ ion was obtained from the tandem accelerator using the terminal ECR ion source [13]. The primary $\mathrm{Ar}^{6+}$ or post-stripped $\mathrm{Ar}^{\mathrm{q}+}(\mathrm{q}=13-15)$ ions were guided into the target foils inside a collision chamber kept at the base pressure of $10^{-8}$ Torr and finally collected at a Faraday cup. Electrons emitted from the projectile ions passing through self-supported C-foil targets of $4.0-20 \mu \mathrm{g} / \mathrm{cm}^{2}$ were energy-analyzed with a tandem electrostatic analyzer located at zero degrees with respect to the ion beam direction. The electron-pass energy for the second analyzer was set at 50 $\mathrm{eV}$ to improve the resolution and also to keep its transmission constant. Electron spectra in the laboratory frame were obtained by scanning the retarding potential between the first and the second analyzers with 1 or $0.5 \mathrm{eV}$ steps for a fixed integrated ion beam current at the Faraday cup. The observed electron spectra were dominated by the so-called cusp peak at around $1.11 \mathrm{keV}$ and a series of Coster-Kronig electron peaks was superposed on the low and high energy wings of the cusp, corresponding to the backward and forward ejected electrons, respectively, from the moving projectile (see Fig. 1(a) or Fig. 1 of the reference [11]). Two spectra for the backward and forward ejected electrons were obtained by converting data points into the projectile rest frame from the laboratory frame (Bw and Fw spectra (raw) in Fig. 1(b)). This conversion resulted in the energy resolution of $\sim 0.3 \mathrm{eV}$ for the electron energy region of our interest $(\sim 15 \mathrm{eV})$ including the spectrometer's resolution. The spectrum from the high energy wing has an almost constant background which comes from a part of the cusp electrons, meanwhile the low energy wing spectrum has an additional background coming from soft collisions. After subtracting these backgrounds, these two spectra have been found to be practically the same in peak energies and intensity distributions (Bw and Fw spectra (bg) in the Fig. 1(b)), showing that the transmission of our electron analyzer system remains constant in the present energy region. The combination of the projectile charge states and the target foil thicknesses studied are shown in table I with the precise centroid energy values of the cusp.

\section{RESULTS AND DISCUSSION}

The beam current in the Faraday cup is found to be the same within $10 \%$ uncertainty in switching the target foil thickness for all the charge states investigated. Our separate measurement of the charge state distribution and mean charge state for $2.0 \mathrm{MeV} / \mathrm{u} \mathrm{S}^{6+}$ ion through carbon foil target [14] shows that the projectile mean charge state rapidly grows until $5 \mu \mathrm{g} / \mathrm{cm}^{2}$
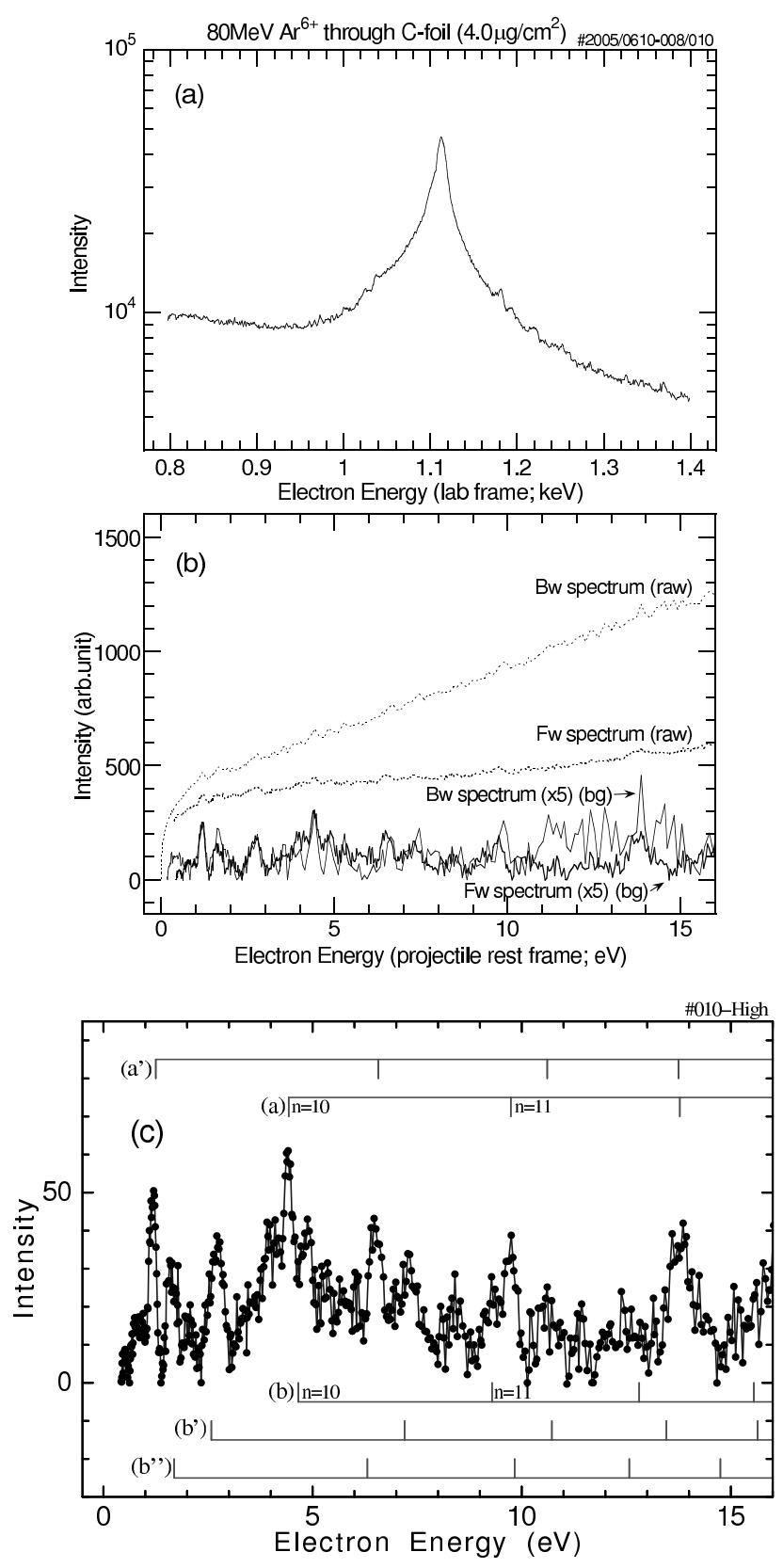

FIG. 1: (a) Electron energy spectrum in the laboratory frame for 80 $\mathrm{MeV}(2.0 \mathrm{MeV} / \mathrm{u}) \mathrm{Ar}^{6+}$ ion passing through a $4.0 \mu \mathrm{g} / \mathrm{cm}^{2}$ carbonfoil measured at zero degrees. (b) Electron energy spectra transformed into the projectile rest frame. Thin dotted curves correspond to the energy spectra from the forward and backward (denoted $\mathrm{Fw}$ and $\mathrm{Bw}$, respectively) ejections, and the solid curves are background-subtracted spectra. (c) Background-subtracted electron energy spectrum ejected at zero-degrees from $80 \mathrm{MeV} \mathrm{Ar}^{6+}$ ion transmitted through $4.0 \mu \mathrm{g} / \mathrm{cm}^{2}$ carbon-foil. Energy refers to the projectile rest frame. Five series of repetitive peaks are assigned to originate from Coster-Kronig transitions $\mathrm{Ar}^{14+} 1 \mathrm{~s}^{2} 2 \mathrm{p}\left({ }^{2} \mathrm{P}_{3 / 2,1 / 2}\right) n l$ $\left.1 \mathrm{~s}^{2} 2 \mathrm{~s}^{2} \mathrm{~S}_{1 / 2}\right) \varepsilon l^{\prime}$ for (a), (a') and $\mathrm{Ar}^{13+} 1 \mathrm{~s}^{2} 2 \mathrm{~s} 2 \mathrm{p}\left({ }^{3} \mathrm{P}_{2,1,0}\right) n l-1 \mathrm{~s}^{2} 2 \mathrm{~s}^{2}$ $\left({ }^{1} \mathrm{~S}_{0}\right) \varepsilon l^{\prime}$ for (b), (b'), (b"), respectively, as described in the text. 


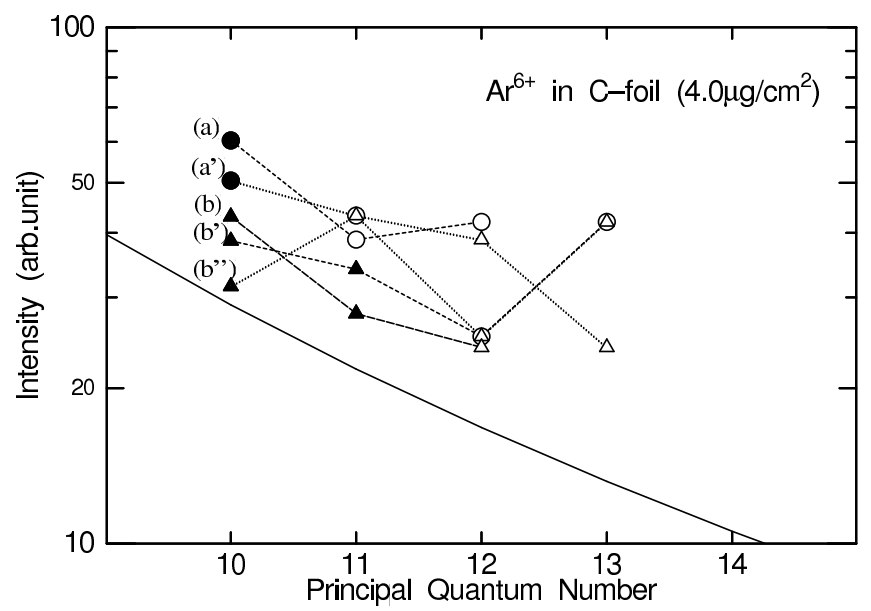

FIG. 2: Dependence of the Coster-Kronig peak intensity on the principal quantum number $n$ of the Rydberg electron emitted from 2.0 $\mathrm{MeV} / \mathrm{u} \mathrm{Ar}{ }^{6+}$ ion penetrating through a $4.0 \mu \mathrm{g} / \mathrm{cm}^{2}$ carbon foil. Full symbols denote well-separated peak intensities, while open symbols denote combined ones. Marks (a), (a'), (b), (b') and (b') denote Coster-Kronig transition series as indicated in the text and Fig. 1(c). Full line shows the $n^{-3}$ dependence.

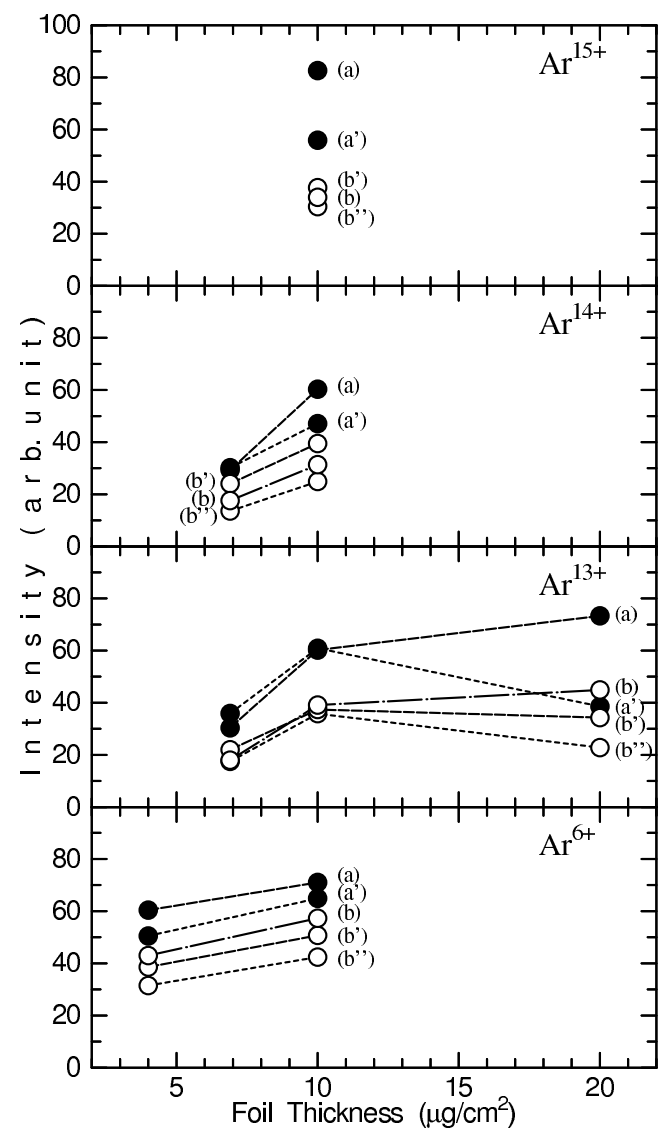

FIG. 3: Coster-Kronig peak intensity of $n=10$ transitions after background subtraction. Marks (a), (a'), (b), (b') and (b") denote C-K transition series as indicated in the text and in Fig. 1(c).
TABLE I: The combination of the projectile charge states and the target foil thicknesses investigated in the present work. Also tabulated are the centroid energy values of the cusp for each measurement. The data with the same symbols (daggers, double daggers and section symbols) were obtained within the same machine time period.

\begin{tabular}{ccc}
\hline $\begin{array}{c}\text { Projectile } \\
\text { Charge State }\end{array}$ & $\begin{array}{c}\text { Target Foil Centroid Energy } \\
\text { Thickness } \\
\left(\mu \mathrm{g} / \mathrm{cm}^{2}\right)\end{array}$ & $\begin{array}{c}\text { of the Cusp } \\
(\mathrm{keV})\end{array}$ \\
\hline $6+$ & 4.0 & $1.1086^{\dagger}$ \\
$6+$ & 10 & $1.1070^{\dagger}$ \\
$13+$ & 6.9 & $1.1018^{\ddagger}$ \\
$13+$ & 10 & $1.1030^{\S}$ \\
$13+$ & 20 & $1.1015^{\S}$ \\
$14+$ & 6.9 & $1.1026^{\ddagger}$ \\
$14+$ & 10 & $1.1037^{\S}$ \\
$15+$ & 10 & $1.1030^{\S}$ \\
\hline
\end{tabular}

and then attains equilibrium values. The same beam current after foil penetration indicates that the projectile mean charge state reaches an equilibrium value within all target thicknesses studied even for the $\mathrm{Ar}^{6+}$ projectile. We therefore assume that the measured electron spectra in the laboratory frame and the derived projectile rest frame (P-frame) spectra can be normalized to within $10 \%$ by the integrated Faraday cup currents, regardless of the projectile charge state or the foil thickness. In Fig. 1(c) the high energy wing of the electron energy spectra is shown, converted into the P-frame and background subtracted, from 2.0 MeV/u $\mathrm{Ar}^{6+}$ traveling through a carbon foil of $4.0 \mu \mathrm{g} / \mathrm{cm}^{2}$ in thickness. The repetitive peaks are assigned to originate from 5 series of Coster-Kronig $(\mathrm{C}-\mathrm{K})$ transitions, indicated by the vertical bars marked (a), (a'), (b), (b') and (b") in the figure. Each series comes from transitions of

(a) $\mathrm{Ar}^{14+} 1 \mathrm{~s}^{2} 2 \mathrm{p}\left({ }^{2} \mathrm{P}_{3 / 2}^{\mathrm{o}}\right) n l-1 \mathrm{~s}^{2} 2 \mathrm{~s}\left({ }^{2} \mathrm{~S}_{1 / 2}\right) \varepsilon l^{\prime}$,

(a') $\mathrm{Ar}^{14+} 1 \mathrm{~s}^{2} 2 \mathrm{p}\left({ }^{2} \mathrm{P}_{1 / 2}^{\mathrm{o}}\right) n l-1 \mathrm{~s}^{2} 2 \mathrm{~s}\left({ }^{2} \mathrm{~S}_{1 / 2}\right) \varepsilon l^{\prime}$,

(b) $\mathrm{Ar}^{13+} 1 \mathrm{~s}^{2} 2 \mathrm{~s} 2 \mathrm{p}\left({ }^{3} \mathrm{P}_{2}^{\mathrm{o}}\right) n l-1 \mathrm{~s}^{2} 2 \mathrm{~s}^{2}\left({ }^{1} \mathrm{~S}_{0}\right) \varepsilon l^{\prime}$,

(b') $\mathrm{Ar}^{13+} 1 \mathrm{~s}^{2} 2 \mathrm{~s} 2 \mathrm{p}\left({ }^{3} \mathrm{P}_{1}^{\mathrm{o}}\right) n l-1 \mathrm{~s}^{2} 2 \mathrm{~s}^{2}\left({ }^{1} \mathrm{~S}_{0}\right) \varepsilon l^{\prime}$,

(b") $\mathrm{Ar}^{13+} 1 \mathrm{~s}^{2} 2 \mathrm{~s} 2 \mathrm{p}\left({ }^{3} \mathrm{P}_{0}^{\mathrm{o}}\right) n l-1 \mathrm{~s}^{2} 2 \mathrm{~s}^{2}\left({ }^{1} \mathrm{~S}_{0}\right) \varepsilon l^{\prime}$,

where the principal quantum number, $n$, of the Rydberg electrons starts at 10 and increases with increasing peak energy for each series. The energy values in parenthesis give the series limits. The marked energies are obtained with the formula

$$
E=\Delta E-Q^{2} R y / 2 n^{2}
$$

where $\Delta E$ is the energy level of the initial core configuration with respect to the final (ground) configuration, $Q$ is the effective charge of the core ion configuration (assumed to be +15 and +14 for (a) and (b) series, respectively), and $R y / 2$ is the Rydberg energy (13.6 eV). For $\Delta E$, compiled level data for $\mathrm{Ar}$ XV and Ar XVI taken from "NIST Atomic Spectra Database" [15] are used. Though not shown here, all the P-frame spectra 
taken from the high energy wings of the 8 measured electron spectra tabulated in the table I also show significant contributions from the $5 \mathrm{C}-\mathrm{K}$ transitions identified above. However, the spectra also show the presence of a few further peaks which remain unassigned within the present investigated energy range. These assignments indicate that at least $\mathrm{Ar}^{15+}$ $1 \mathrm{~s}^{2} 2 \mathrm{p}\left({ }^{2} \mathrm{P}\right)$ and $\mathrm{Ar}^{14+} 1 \mathrm{~s}^{2} 2 \mathrm{~s} 2 \mathrm{p}\left({ }^{3} \mathrm{P}\right)$ states play an important role for argon ion in penetrating through carbon foils in near equilibrium thickness region.

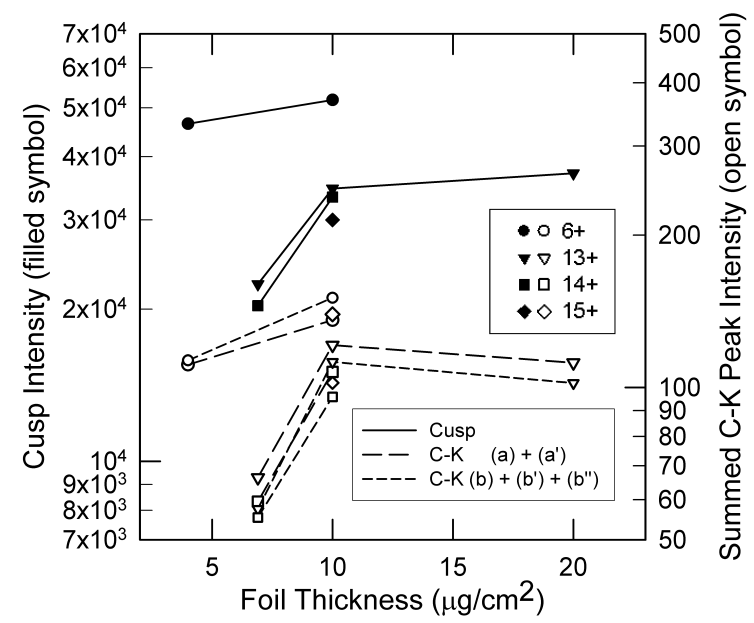

FIG. 4: Cusp electron intensity (left-side axis; full symbols) and summed Coster-Kronig electron peak intensity (right-hand axis; open symbols) for $\mathrm{Ar}^{6,13,14,15+}$ ions after penetrating carbon foil targets of $4.0-20 \mu \mathrm{g} / \mathrm{cm}^{2}$. Full line denotes cusp intensity, and long and short dashed lines denote summed Coster-Kronig peak intensity for the initial states of $\mathrm{Ar}^{14+} 1 \mathrm{~s}^{2} 2 \mathrm{p}\left({ }^{2} \mathrm{P}\right) 10 l\left((\mathrm{a})+\left(\mathrm{a}^{\prime}\right)\right.$ in the text for $n=10)$ and $\mathrm{Ar}^{13+} 1 \mathrm{~s}^{2} 2 \mathrm{~s} 2 \mathrm{p}\left({ }^{3} \mathrm{P}\right) 10 l\left((\mathrm{~b})+\left(\mathrm{b}^{\prime}\right)+\left(\mathrm{b}^{\prime \prime}\right)\right.$ for $\left.n=10\right)$, respectively.

These repetitive peaks of $\mathrm{C}-\mathrm{K}$ electrons are found to follow the $n^{-3}$ law, as illustrated in Fig. 2, as has been pointed out by Breinig et al. [16]. Taking into account that some very closely located C-K peaks appear as a combined peak, such as $n=11$ of (a') and $n=11$ of (b"), well-separated peaks and appropriate division of combined peak heights lead us to the $n^{-3}$ law. It can be seen in Figs. 1(c) and 2 that the $n=10$ peaks are wellseparated for all the $5 \mathrm{C}$-K series and their peak height can be an index to describe the collision phenomena. From this point of view, the $n=10$ peak heights taken from the (background subtracted) P-frame spectra are plotted in Fig. 3 versus the foil thickness. According to the three-step transport model, the population of the Rydberg state is ruled both by the population of appropriate ion core state(s) and the intensity of the near-threshold continuum electrons traveling within the solid alongside the projectile (the entrained electrons), because the number of the entrained electrons is not large enough to form the Rydberg state on every possible occasion. It is clear that the number of the entrained electrons is strongly correlated with the cusp electron yields. Fig. 4 plots cusp electron intensity measured in the laboratory frame (Fig. 1(a)) and summed

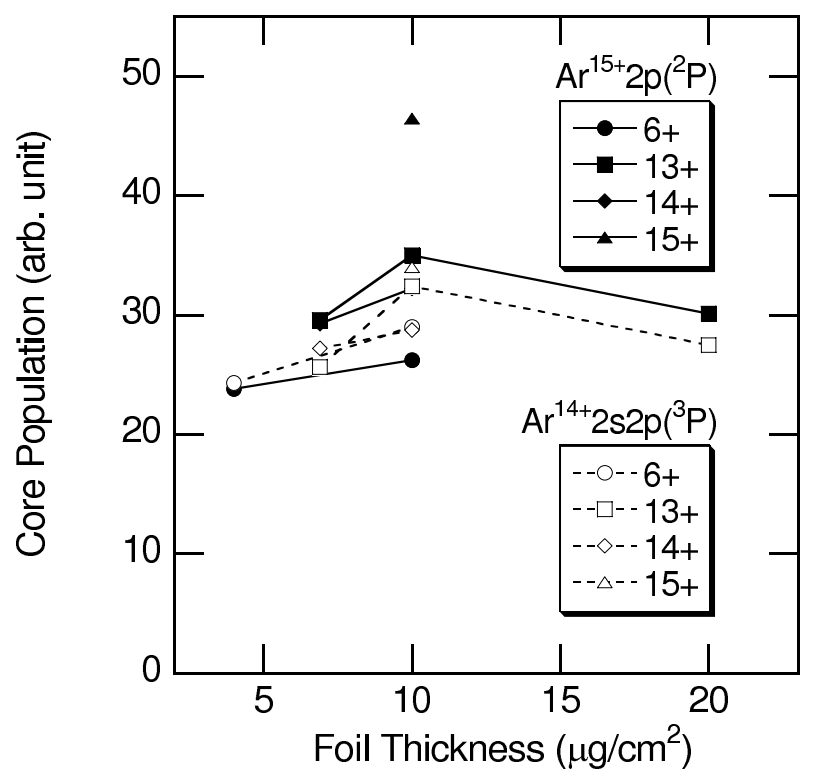

FIG. 5: Population of $\mathrm{Ar}^{15+} 1 \mathrm{~s}^{2} 2 \mathrm{p}\left({ }^{2} \mathrm{P}\right)$ and $\mathrm{Ar}^{14+} 1 \mathrm{~s}^{2} 2 \mathrm{~s} 2 \mathrm{p}\left({ }^{3} \mathrm{P}\right)$ states derived by the intensities of Coster-Kronig and cusp electron peaks for $\mathrm{Ar}^{6,13,14,15+}$ ions after penetrating carbon foil targets of $4.0-20$ $\mu \mathrm{g} / \mathrm{cm}^{2}$.

intensities of $n=10 \mathrm{C}-\mathrm{K}$ peaks over different $J$ values of the $\mathrm{Ar}^{15+}$ and $\mathrm{Ar}^{14+}$ cores (for (a)+(a') and (b)+(b')+(b"), respectively) as functions of the foil thickness. The $\mathrm{C}-\mathrm{K}$ peak intensity for each initial charge state was found to show very good agreement with the cusp intensity. These facts imply that the entrained electron intensity exerts a major influence compared to the ion core population on the Rydberg peak intensity, and the $\mathrm{Ar}^{15+} 1 \mathrm{~s}^{2} 2 \mathrm{p}\left({ }^{2} \mathrm{P}\right)$ and $\mathrm{Ar}^{14+} 1 \mathrm{~s}^{2} 2 \mathrm{~s} 2 \mathrm{p}\left({ }^{3} \mathrm{P}\right)$ ion core populations seem to take nearly the same values except for $\mathrm{Ar}^{15+}$ core for $\mathrm{Ar}^{15+}$ incidence (Fig. 5). This result is consistent with our separate measurement of charge fractions of $2.0 \mathrm{MeV} / \mathrm{u} \mathrm{S}$ ions penetrating carbon foils [14], which concluded that the processes involving L-shell electrons are equilibrated within the target thickness of $\sim 5 \mu \mathrm{g} / \mathrm{cm}^{2}$ and the charge equilibration beyond this thickness is ruled by $\mathrm{K}$-shell processes. Comparison of the peak intensities of (a) and (a') for $\mathrm{Ar}^{13+}$ projectile at foil thickness 10 and $20 \mu \mathrm{g} / \mathrm{cm}^{2}$ (Fig. 3) gave a tentative scenario that the spin quantum number of the L-shell electrons $(J$ for coupled states) increased during the continual excitation of the electrons through collisions with the target electrons. The total intensity of (a) and (a') were found to remain constant as the target thickness increased from 10 to $20 \mu \mathrm{g} / \mathrm{cm}^{2}$ as did the total and relative intensities of (b), (b') and (b").

In the present case of $\mathrm{Ar}^{14+}$ and $\mathrm{Ar}^{13+}$ incident ions, the summed intensities of (a) and (a') $\mathrm{C}-\mathrm{K}$ peaks of $\mathrm{Ar}^{14+}$ are found to be nearly the same as the corresponding states (b), (b') and (b") of $\mathrm{Ar}^{13+}$, and so do the $\mathrm{Ar}^{15+}$ and $\mathrm{Ar}^{14+}$ core populations, as illustrated in Figs. 4 and 5 , respectively. This suggests that the $\mathrm{Ar}^{15+} 1 \mathrm{~s}^{2} 2 \mathrm{p}\left({ }^{2} \mathrm{P}\right)$ and $\mathrm{Ar}{ }^{14+} 1 \mathrm{~s}^{2} 2 \mathrm{~s} 2 \mathrm{p}\left({ }^{3} \mathrm{P}\right)$ states have nearly the same population in the solid near or after their equilibration. Furthermore, the equilibrium $\mathrm{Ar}^{14+}$ fraction is found to be almost twice as large as $\mathrm{Ar}^{15+}$ frac- 
tion after exiting the foil target [17]. This would constitute another qualitative proof that the projectile charge states shift downwards upon exiting the solid.
[1] J. Rothermal, H.-D. Betz, F. Bell, and V. Zacek, Nucl. Instr. and Meth 194, 341 (1982).

[2] H.-D. Betz, J. Rothermal, D. Röschenthaler, F. Bell, R. Schuch, and G. Nolte, Phys. Lett. 91A, 12 (1982).

[3] Y. Yamazaki et al., Phys. Rev. Lett. 61, 2913 (1988).

[4] J. Burgdörfer and C. Bottcher, Phys. Rev. Lett. 61, 2917 (1988)

[5] N. Stolterfoht, Phys. Rep. 146, 315 (1987).

[6] A. Itoh, T. Schneider, G. Schiwietz, Z. Roller, H. Platten, G. Nolte, D. Schneider, and N. Stolterfoht, J. Phys. B 16, 3965 (1983).

[7] K. Kawatsura et al., Nucl. Instr. and Meth. B 53, 421 (1991).

[8] M. Imai, M. Sataka, Y. Yamazaki, K. Komaki, K. Kawatsura, and Y. Kanai, Physica Scripta T73, 93 (1997).

[9] M. Sataka, M. Imai, K. Kawatsura, K. Komaki, H. Tawara, A. Vasilyev, and U. I. Safronova, J. Phys. B 16, 267 (2002).

[10] M. Sataka, M. Imai, K. Kawatsura, K. Komaki, H. Tawara, A. Vasilyev, and U. I. Safronova, Phys. Rev. A 65, 052704 (2002).

[11] M. Imai, M. Sataka, S. Kitazawa, K. Komaki, K. Kawatsura, H. Shibata, H. Tawara, T. Azuma, Y. Kanai, and Y. Yamazaki, Nucl. Instr. and Meth. B 193, 674 (2002).
[12] K. Kawatsura, K. Takahiro, M. Imai, M. Sataka, K. Komaki, and H. Shibata, Nucl. Instr. and Meth. B 205, 528 (2003).

[13] M. Matsuda, C. Kobayashi, and S. Takeuchi, in: K. W. Shepard (Ed.), Heavy Ion Accelerator Technology, AIP, New York, 1999, p. 65 (1999).

[14] M. Imai, M. Sataka, K. Kawatsura, K. Takahiro, K. Komaki, H. Shibata, H. Sugai, and K. Nishio, Nucl. Instr. and Meth. B 230, 63 (2005).

[15] Available online at http://physics.nist.gov/PhysRefData/ASD/index.html. for details, Yu Ralchenko, J. R. Fuhr, F.-C. Jou, A. E. Kramida, W. C. Martin, L. I. Podobedova, J. Reader, E. B. Saloman, J. E. Sansonetti, and W. L. Wiese, in: T. Kato, H. Funaba, D. Kato (Ed.), Atomic and Molecular Data and Their Applications, AIP, New York, 2005, p. 276 (2005).

[16] M. Breinig et al., Phys. Rev. A 25, 3015 (1982).

[17] K. Shima, N. Kuno, M. Yamanouchi, and H. Tawara, At. Data Nucl. Data Tables 51, 73 (1992). 